\title{
Fontes de informação como valor agregado ao sistema eletrônico de revistas da Universidade Federal do Paraná (OJS/SER-UFPR)
}

\author{
Patrícia Zeni Marchiori* \\ André Luiz Appel $^{* *}$
}

\begin{abstract}
Resumo Apresenta proposta para agregar valor ao Sistema Eletrônico de Revistas da Universidade Federal do Paraná (OJS/SER/UFPR) a partir da exploração da estrutura e conteúdo da Research Support Tool (RST). Descreve os avanços obtidos até o momento, concentrados na elaboração de um diagrama de dimensões de qualidade para fontes de informação; na tradução dos termos do inglês para o português; na avaliação para permanência e descarte de fontes de informação default da ferramenta; e no modelo de resumo informativo das fontes a serem mantidas ou inseridas no Sistema. Discute os problemas recorrentes relativos às fontes de informação e sua recuperação, assim como as questões que surgem a partir de uma tecnologia de comunicação de ponta.
\end{abstract}

Palavras-chave Metodologias em Gestão da Informação; Humanidades - Fontes de Informação; Fontes de informação - critérios de qualidade; Sistema Eletrônico de Revistas - Research Support Tool; Fontes de informação - descrição.

\section{Sources of information as value added to the electronic journals system of the Federal University of Paraná}

\begin{abstract}
This article presents a proposal for a value-added Research Support Tool (RST), which is part of the Open Journal System (OJS). It describes the proposal's achievements so far, such as: a multidimensional information sources quality criteria framework; the RST/Context terms in translation to Portuguese; evaluation of the default sources; and an abstract template for the sources to be maintained or incorporated into the System. It discusses the recurrent problems concerning information sources and information retrieval, as well as the questions arising from cutting-edge communication technology.
\end{abstract}

Keywords Information Research Management methodologies; Information Sources Humanities; Information Sources - quality criteria; Open Journal System - Research Support Tool ; Information Sources - description.

\footnotetext{
*Professor Adjunto IV do Departamento de Ciência e Gestão da Informação da UFPR, especialista em Gestão do Conhecimento em Ciências da Água, Hidrologia e Oceanografia (VUB/Bélgica), Mestre em Ciência da Informação (UFRJ/IBICT) e Doutora em Comunicação (ECA/USP). R: Lothário Meissner, 632 - Jardim Botânico Curitiba/Paraná - CEP 80210-170, Tel. (0x41) 3360-4421, email: pzeni @ufpr.br

** Graduando em Gestão da Informação pela Universidade Federal do Paraná. Bolsista voluntário no Programa de Iniciação Científica UFPR/CNPq (IC-Voluntária/UFPR/CNPq), R: Lothário Meissner, 632 - Jardim Botânico Curitiba/Paraná, Tel. (0x41) 9645-5797, email: appel@ufpr.br
} 


\section{Introdução e estrutura metodológica da pesquisa em andamento}

Dentre os diferentes vetores que compõem o sistema de comunicação científica, os periódicos acadêmicos têm sido um dos mais afetados pelos recentes avanços tecnológicos, cujas potencialidades contribuem para a revisão do modelo tradicional de geração/consumo de informação científica. As discussões em torno dos movimentos e iniciativas de acesso aberto polarizam opiniões ao mesmo tempo em que, abstraídas todas as resistências, oferecem vantagens comprovadas ao processo de análise e recuperação de informação. Extrapolando-se a dimensão político-ideológica, o modelo proposto fundamenta-se em uma tecnologia de custos reduzidos, auto-sustentável, voltada para a interoperabilidade por meio do uso de padrões de metadados de complexidade relativamente baixa. A criação coletiva e compartilhada de ferramentas de acesso livre tem se materializado em aplicativos, entre os quais se pode citar o Open Conference System (OCS)/Sistema Online de Apoio a Congressos (SOAC) e o Open Journal Systems (OJS)/Sistema Eletrônico de Editoração de Revistas (SEER) (PUBLIC Knowledge Project, 2007).

Destas ferramentas disponíveis, traduzidas para o português pelo Instituto Brasileiro de Ciência e Tecnologia (IBICT), o OJS/SEER vem ampliando sua carteira de usuários que, paulatinamente, reconhecem o conjunto de características aliadas ao sistema de gerenciamento de fluxo editorial. Incluem-se nestas características tanto aquelas relativas ao uso de protocolos de metadados, quanto às possibilidades de recuperação via provedores de serviço e de preservação digital. Porém, na medida em que a plataforma de usuários se expande, o mesmo ocorre com a exploração de outras funcionalidades do aplicativo, como, por exemplo, a finalidade e utilidade da Research Support Tool (RST).

De forma a contribuir com a comunidade envolvida no desenvolvimento e no fortalecimento dos aplicativos de plataforma livre, descreve-se o andamento e resultados alcançados até o momento no projeto de pesquisa intitulado "Fontes de informação como valor agregado ao Sistema Eletrônico de Revistas (SER) da UFPR", que tem como objetivos:

a) Investigar as potencialidades e limitações da RST do OJS/SER/UFPR, definindo uma estrutura de tradução de termos e inserção de fontes para o link 'context';

b) Alterar a RST/Context, inserindo fontes de informação específicas para cada item;

c) Criar uma revista piloto na área de Comunicação Social como plataforma para a inserção de fontes internacionais e nacionais, com base em padrões de qualidade previamente definidos;

d) Verificar a possibilidade de expandir a metodologia de trabalho para as demais revistas na área de Comunicação, em um primeiro momento, e para outras quando da avaliação e ajustes do projeto.

Este artigo apresentará resultados parciais dos itens a, b e c com base na proposta metodológica abaixo, cujo detalhamento ocorrerá oportunamente ao longo do texto:

a) Levantamento na literatura das áreas de Biblioteconomia, Ciência da Informação e correlatas, visando levantar os critérios confiáveis para a avaliação de fontes de informação; 
b) Coleta de informação adicional sobre o OJS/SER/UFPR e, mais especificamente, sobre $\mathrm{RST} /$ Context, verificando a tipologia de fontes, as áreas de conhecimento envolvidas e as fontes disponíveis por default;

c) Alteração/tradução de termos para o português, avaliação das condições de acesso às fontes default e criação de um modelo para a elaboração de resumo indicativo para as fontes que permanecerão ou serão inseridas no Sistema;

d) Definição de uma estratégia de trabalho e formulário para a localização e descrição de novas fontes, seguida da efetiva alteração de elementos do Sistema, a partir da inserção, expansão ou retirada de registros;

e) Teste da nova interface do Sistema (particularmente na RST/Context) e definição de uma metodologia de avaliação da revista piloto.

A opção de estudo da RST/Context justifica-se pela possibilidade de utilizá-la como um laboratório para a avaliação e construção de conteúdos informativos, evidenciando a complementaridade de uma visão não-técnica aos aplicativos de software. Além disto, oportuniza discussões de fundo na área de Ciência da Informação como, por exemplo, a definição e uso de critérios de qualidade para material disponível na web considerando-se a filosofia de construção colaborativa da ferramenta e o uso de recursos livres de custo e copyright.

\section{Extrapolando o uso da RST: uma proposta colaborativa}

Estruturalmente, a RST é uma barra de ferramentas localizada ao lado direito de cada artigo publicado no OJS/SEER (ou SER-UFPR) (FIGURA 1), contendo links de hipertexto que apontam, a partir do artigo, para um conjunto de recursos selecionados. Tais recursos estão relacionados à(s) área(s) de conhecimento onde o periódico se insere, estando disponíveis para a consulta pelo pesquisador, expandindo o acesso a materiais que possam, potencialmente, lhe ser úteis.

Os links em uma RST podem ser categorizados em três camadas (ou seções) identificadas como: 'For this article', 'Context' e 'Action'. 


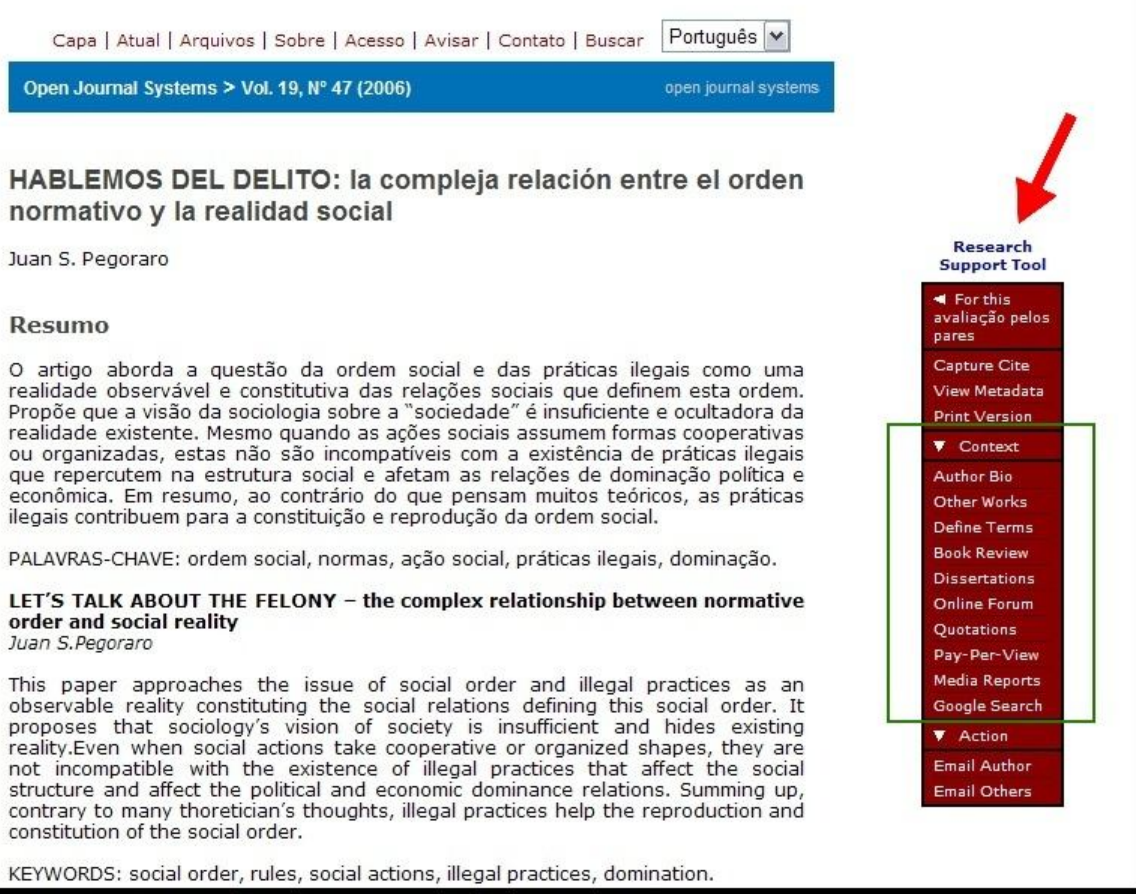

Figura 1 - A Research Support Tool do OJS/SEER

A camada 'For this article' oferece informações sobre a condição formal do artigo indicando: o status e tipo de estudo; sugestão de citação do artigo em diferentes formatos/padrões e software bibliográfico (Procite, entre outros); os metadados referentes à revista como um todo e ao artigo em particular, entre outras possibilidades. A camada 'Action' atua como a funcionalidade interativa de uma RST, pois, a partir dela, os leitores podem se comunicar com os autores, incluir comentários e avisar colegas da existência do artigo, por exemplo.

A camada 'Context' foi construída, assim como as demais, sob a hipótese de que, dado um conjunto estendido de sugestões de outras fontes conectadas ao artigo, agregar-se-ia valor às habilidades de busca e apreensão de informação do pesquisador. Apresenta-se como uma lista expansiva de outras fontes de informação na(s) temática(s) do periódico, possibilitando seu aproveitamento para a expansão de fontes na(s) área(s) de conhecimento específica(s). A tipologia de tais fontes varia, desde bases de dados de acesso aberto até links para material pago. Ao se clicar um dos itens, uma janela pop-up é acionada contendo uma lista de fontes préselecionadas pelo OJS/SEER e que podem - potencialmente - ser modificadas pelo administrador/editor. As palavras-chave de busca são, também por default, inseridas via os metadados definidos pelo autor do artigo e, da mesma forma, podem ser alteradas pelo interessado. A ferramenta é igualmente flexível ao permitir alterações nas diferentes camadas/seções e links da RST, pois seu propósito é atuar como uma ponte entre o artigo de origem e seu contexto mais amplo, poupando o tempo dos pesquisadores em buscas aleatórias.

Alinhada com os princípios de uma ciência 'aberta', a RST - também disponível nas versões recentes do $O C S / \mathrm{SOAC}$ - foi construída com o objetivo de estimular a "polinização cruzada" entre diferentes recursos informativos, em que o criador do conteúdo é menos importante que a intenção e o desejo do leitor (BARTHES, citado por WILLINSKY, 2004). Uma rede crescente 
de recursos interligados não só acomodará um sistema auto-sustentável de alerta corrente de diferentes materiais de cunho científico-acadêmico, estimulando o trabalho colaborativo, como sedimentará o processo de comunicação como instância de vínculo e legitimidade.

Em estudo realizado com 13 formuladores de políticas no Canadá, Willinsky (2004) apresentou a RST como uma alternativa ao acesso às informações de apoio à tomada de decisão. Neste particular, um dos objetivos era o de transportar a RST para além do ambiente acadêmico e, ao mesmo tempo, trazer tais recursos para o universo selecionado. Apoiados neste ambiente inovativo de publicação, segundo o autor, os entrevistados encontrariam condições apropriadas, controladas e mais aprofundadas para a leitura, interpretação e utilização de material de pesquisa. Os resultados, obtidos com o auxílio de entrevista em profundidade, revelaram algumas peculiaridades nos hábitos de pesquisa na Internet, assim como diferenças de comportamento de busca quando comparados àqueles utilizados por pesquisadores e acadêmicos (incluídos aí os jargões e nomenclaturas). Ainda que com um universo restrito, o estudo identificou o crescente interesse dos formuladores de políticas em fontes de acesso livre, ainda que a ferramenta apresente desafios quanto ao design, ao equilíbrio e à intencionalidade das bases de dados face aos objetivos e tarefas de responsabilidade dos entrevistados.

\section{A contribuição das fontes de informação para a comunicação científica}

Em recente artigo, Mueller (2005) afirma que já há um consenso sobre o fato de que pesquisadores de áreas diferentes utilizam distintos canais de comunicação para disponibilizar seus resultados aos pares e demais interessados. Mais especificamente, pesquisadores das Ciências Sociais e das Humanidades defendem

...critérios que respeitem especificidades de cada área, de acordo com temas, abordagens e métodos de pesquisa mais característicos. Argumentam que entre áreas distintas e mesmo dentro de uma única área, as diferenças de enfoque e conteúdo demandam formas de comunicação igualmente diferentes, com características próprias (MUELLER, 2005, f.1).

A autora cita Meadows, ao explorar o fato de que, ainda que os artigos publicados em periódicos científicos sejam tomados como uma medida normalmente usada para avaliar a quantidade de informações provenientes de um pesquisador, “...nem sempre, a quantidade de artigos publicados reflete a produtividade de autores" (MUELLER, 2005, f.1). Neste sentido, pesquisadores das áreas de Ciências Sociais e Humanidades tendem a produzir textos relativamente longos, publicados como capítulos de livros ou mesmo livros no todo. Tal tendência é demonstrada não só nos resultados apresentados pela autora como também no estudo de Juanes et alii (2002), cujo objetivo foi analisar a produção científica em Comunicação dos docentes da ECA/USP. Dentre os resultados obtidos, destacam-se aqueles oriundos do Departamento de Jornalismo e Editoração (CJE) que indicam um maior número de livros publicados quando comparados a publicações em outros canais de comunicação. Possivelmente, refletem os autores, tal situação se

Liinc em Revista, v.4, n.2, setembro 2008, Rio de Janeiro, p. 253-268 - http://www.ibict.br/liinc 
justifica diante "... [d] as demandas de mercado resultantes das teses publicadas nesse formato, a exemplo do que ocorre nas demais áreas das ciências sociais e humanas" (JUANES et alii, 2002, p. 11).

No que diz respeito aos periódicos científicos, Stumpf (2003) avaliou 26 revistas publicadas na área de Comunicação sob o ponto de vista de docente-pesquisadores de 12 Programas de PósGraduação na área. Ainda que os resultados tenham apontado oito revistas como sendo as mais conceituadas, a autora considera que não há - até o momento da pesquisa o que chama de "revista padrão em Comunicação", agravada pelo fato de que dos 26 títulos apresentados, apenas oito foram reconhecidos por mais da metade dos 71 entrevistados.

De qualquer maneira, independentemente do vetor/canal de publicação e divulgação, pode-se dizer que a produção e o consumo de informação científica repousam no acesso a fontes de informação relevantes, tanto para a análise de métodos e pesquisas em desenvolvimento como para a fundamentação e experimentação de novos estudos.

Inúmeras são as dificuldades para a identificação e compilação de fontes de informação nas diferentes áreas do conhecimento. Recorrentemente, Campello (1988, 1998, 2000) aponta para os problemas mais comuns relacionados à forma como pesquisadores localizam e selecionam a informação, quanto:

- ao formato em que as informações são apresentadas, o suporte e a função de tais fontes;

- à explosão bibliográfica e o fato de várias fontes de informação se tornarem obsoletas com maior freqüência que outras;

- às dificuldades de comunicação entre os membros de determinada área científica;

- à barreira do idioma;

- ao custo envolvido na organização, distribuição e manutenção de tais fontes.

Peixoto (2002) corrobora e adiciona outros pontos de impacto, tais como:

- a oferta inadequada dos acervos bibliográficos, principalmente nos aspectos de escassez, custo e desatualização dos materiais;

- a tendência de se preferir o uso de informações obtidas pelos recursos computacionais;

- o fato de que a informação está rapidamente disponível, mas é muito volumosa, extremamente dispersa e de confiabilidade variável.

A confiabilidade parece ser uma preocupação estrutural quando se considera o uso de uma fonte de informação no âmbito acadêmico/científico. É de senso comum que um pesquisador responsável deva considerar critérios de seleção e qualidade para legitimar a consistência e credibilidade das fontes que venha a utilizar. Portanto, ao se propor uma listagem de fontes como valor agregado a um produto de informação, tais critérios devem ser discutidos e explicitados, justificando-se sua escolha. 


\section{Qualidade de fontes de informação}

Recorrentemente, profissionais da informação discutem, testam e implantam metodologias e soluções visando agregar valor à localização e descrição de recursos de informação disponíveis em sistemas, produtos ou serviços de informação. Ao abordarem questões éticas envolvidas na chamada "vida social da informação", Alfino e Pierce (2001) consideraram o advento da Internet como um desafio ao princípio de neutralidade do bibliotecário e dos cientistas da informação. Para estes autores, a diversidade crescente da informação disponível em rede desafia os conceitos de "desenvolvimento de coleções" e dos "serviços de referência" tradicionais. Justificam tal afirmativa ao declarar que os critérios para a seleção de fontes de pesquisa podem "ameaçar" a autonomia dos usuários em relação ao que este considera como válido em termos de conteúdo, design e aplicabilidade/impacto em suas atividades. O próprio conceito de neutralidade não acontece em um vácuo, pois evolui com a interação destes profissionais com o contexto e a cultura dominante. Reconhecer as mudanças é provocar interferências nestas mesmas mudanças de modo que a sociedade continue a respeitar e reconhecer o papel destes profissionais em um cenário de produção, oferta e consumo imediato de informação francamente aberto e não-mediado. Porém, ainda que o acesso à Internet amplie as fronteiras de recuperação da informação para além dos acervos construídos e controlados a partir de políticas de desenvolvimento de coleções e critérios formais de qualidade, permanece a necessidade de se reconhecer o valor existente em dada informação.

O próprio OJS/SER oferece uma lista default de fontes online acessíveis via a RST/Context que, segundo Willinsky (2003), foram selecionadas considerando suas características de acesso livre. Além disto, reforça a possibilidade da RST, como um todo, atuar como elemento catalisador da potencial intenção do autor em expandir sua pesquisa, reforçando a percepção de que nenhum trabalho científico existe de forma isolada. Embora Willinsky (2003, p. 10) declare que a RST deve disponibilizar "material relevante", não há critérios explícitos de seleção das fontes arroladas. Tal fato pode ser creditado à condição de construção colaborativa da ferramenta, apoiada pela possibilidade de que qualquer interessado pode sugerir uma fonte/recurso, preenchendo o formulário disponível em <http://pkp.sfu.ca/recommend_rt>. Neste, a sugestão deve ser seguida de uma descrição da razão pela qual a fonte é considerada como um "bom" recurso a ser inserido na ferramenta.

Portanto, a partir do reconhecimento de que há flexibilidade para a inserção ou retirada de fontes de informação da RST/Context, o Projeto de pesquisa confirmou sua proposta de agregar valor à ferramenta, discutindo e construindo critérios de qualidade para sua seleção. Considerando-se que parte significativa dos recursos a serem agregados à RST/Context existe apenas em forma digital, analisaram-se roteiros selecionados (KIRK, 1996; BIREME, 2005; TILLMAN, 2003; ORMONDROYD, 2004; RIEH, BELKIN, 1998; RIEH, BELKIN, 2000, RIEH, 2005) finalizando-se uma estrutura básica de critérios explicitada na Figura 2.

Liinc em Revista, v.4, n.2, setembro 2008, Rio de Janeiro, p. 253-268 - http://www.ibict.br/liinc 


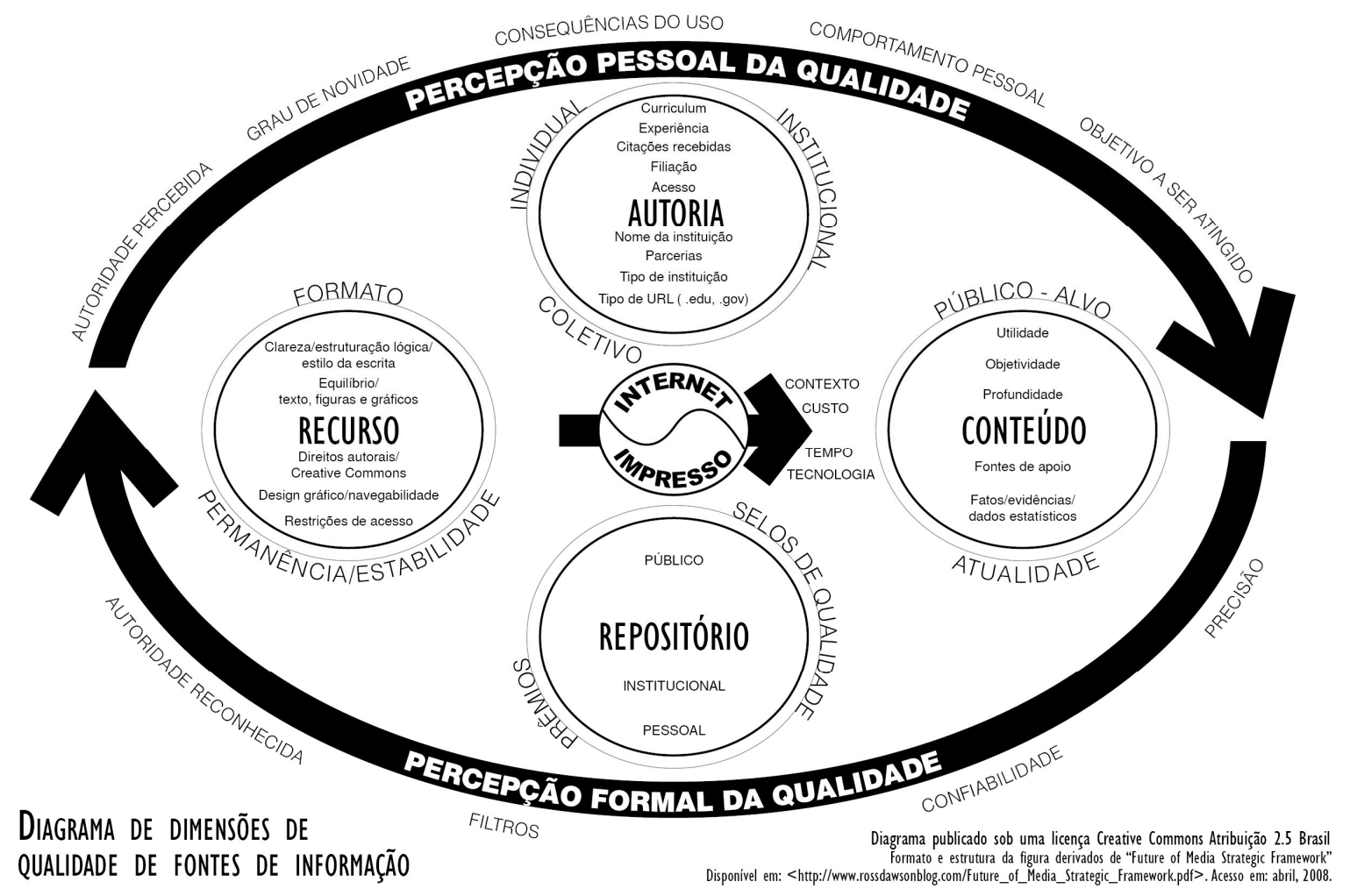

Figura 2 - Diagrama de Dimensões de Qualidade de Fontes de Informação

Fonte: os autores ${ }^{1}$

A idéia que sustenta esta estrutura consiste na flexibilização de critérios tradicionalmente utilizados para a avaliação de fontes de informação, uma vez que a percepção pessoal da qualidade pode, ainda que não obrigatoriamente, acompanhar a percepção formal da qualidade. Da mesma forma, não há pesos nem hierarquias entre quaisquer das esferas/dimensões e variáveis indicadas, o que permite ao interessado utilizar o conjunto de elementos, adequando-o ao seu ambiente, objetivos e capacidade/experiência de julgamento. Pretende-se, na continuidade do Projeto, aperfeiçoar e discutir mais profundamente as dimensões declaradas nesta primeira versão, aplicando-as na RST/Context, conforme descrito abaixo.

\section{Avaliação e adaptação da $R S T /$ Context - Aspectos funcionais}

Em um primeiro momento construiu-se um "mapa de acesso" o qual permitiu que a equipe de trabalho compreendesse as funcionalidades do sistema, por meio da completa visualização dos

\footnotetext{
1 Formato e estrutura da figura derivada de "Future of Media Strategic Framework". Disponível em: http://www.rossdawsonblog.com/Future_of_Media_Strategic_Framework.pdf. Acesso em: abril, 2008.
} 
possíveis pontos de adaptação da RST/Context.

A camada Context da RST apresenta, por default, uma extensa lista de tipos de categorias de fontes/Contextos distribuídas de acordo com dezenove áreas do conhecimento previamente definidas. Cada contexto agrega um número variável de fontes de informação que define se um determinado contexto aparecerá em diferentes áreas do conhecimento ou ficará restrito a uma área específica. Após levantamento dos contextos nestas áreas, estes foram classificados conforme o número de ocorrências, considerando-se a linha de corte abaixo de dez ocorrências. (Figura 3).

\begin{tabular}{|c|c|c|c|c|c|c|c|c|c|c|c|c|c|c|c|c|c|c|c|c|}
\hline \multirow[b]{2}{*}{$\begin{array}{c}\text { Contextos I } \\
\text { Categorias de Fontes }\end{array}$} & \multicolumn{19}{|c|}{ Áreas do Conhecimento } & \multirow[b]{2}{*}{ 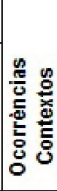 } \\
\hline & 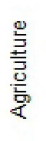 & 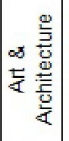 & 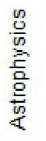 & $\begin{array}{l}\text { 产 } \\
\frac{\circ}{0}\end{array}$ & 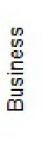 & 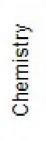 & 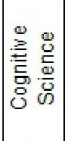 & 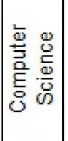 & 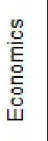 & 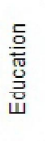 & 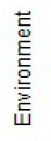 & 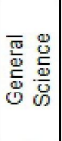 & $\begin{array}{l}\text { 음 } \\
\text { 忢 } \\
0 \\
0\end{array}$ & $\begin{array}{l}\text { 吕 } \\
\stackrel{\underline{\underline{E}}}{\bar{W}} \\
\text { 壱 } \\
\text { 至 }\end{array}$ & 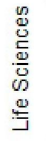 & 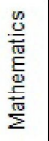 & $\frac{\frac{0}{0}}{\frac{0}{2}}$ & $\begin{array}{l}\frac{0}{0} \\
\stackrel{0}{0} \\
\frac{5}{\alpha}\end{array}$ & 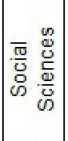 & \\
\hline Author's work's & $\mathrm{x}$ & $\mathrm{x}$ & $\mathrm{x}$ & $\mathrm{x}$ & $\mathrm{x}$ & $\mathrm{x}$ & $\mathrm{x}$ & $\mathrm{x}$ & $\mathrm{x}$ & $\mathrm{x}$ & $\mathrm{x}$ & $\mathrm{x}$ & $\mathrm{x}$ & $\mathrm{x}$ & $\mathbf{x}$ & $\mathrm{x}$ & $\mathrm{x}$ & $\mathrm{x}$ & $\mathrm{x}$ & 19 \\
\hline \begin{tabular}{|l|} 
Media reports \\
\end{tabular} & $\mathrm{x}$ & $\mathrm{x}$ & $\mathrm{x}$ & $\mathrm{x}$ & $\mathrm{x}$ & $\mathrm{x}$ & $\mathrm{x}$ & $\mathrm{x}$ & $\mathrm{x}$ & $\mathrm{x}$ & $\mathrm{x}$ & $\mathrm{x}$ & $\mathrm{x}$ & $\mathrm{x}$ & $\mathrm{x}$ & $\mathrm{x}$ & $\mathrm{x}$ & $\mathrm{x}$ & $\mathrm{x}$ & 19 \\
\hline Online forums & $\mathrm{x}$ & $\mathrm{x}$ & $\mathrm{x}$ & $\mathrm{x}$ & $\mathrm{x}$ & $\mathrm{x}$ & $\mathrm{x}$ & $\mathrm{x}$ & $\mathrm{x}$ & $\mathrm{x}$ & $\mathrm{x}$ & $\mathrm{x}$ & $\mathrm{x}$ & $\mathrm{x}$ & $\mathrm{x}$ & $\mathrm{x}$ & $\mathrm{x}$ & $\mathrm{x}$ & $\mathrm{x}$ & 19 \\
\hline Pay-per-view & $\mathrm{x}$ & $\mathrm{x}$ & $\mathrm{x}$ & $\mathrm{x}$ & $\mathrm{x}$ & $\mathrm{x}$ & $\mathrm{x}$ & $\mathrm{x}$ & $\mathrm{x}$ & $\mathrm{x}$ & $\mathrm{x}$ & $\mathrm{x}$ & $\mathrm{x}$ & $\mathrm{x}$ & $\mathrm{x}$ & $\mathrm{x}$ & $\mathrm{x}$ & $\mathrm{x}$ & $\mathrm{x}$ & 19 \\
\hline Government policy & & $\mathrm{x}$ & $\mathrm{x}$ & $\mathrm{x}$ & $\mathrm{x}$ & $\mathrm{x}$ & $\mathrm{x}$ & $\mathrm{x}$ & $\mathrm{x}$ & $\mathrm{x}$ & $\mathrm{x}$ & $\mathrm{x}$ & $\mathrm{x}$ & $\mathrm{x}$ & $\mathbf{x}$ & $\mathrm{x}$ & $\mathrm{x}$ & $\mathrm{x}$ & $\mathrm{x}$ & 18 \\
\hline Related studies & $x$ & $\mathrm{x}$ & $\mathrm{x}$ & $\mathrm{x}$ & $\mathrm{x}$ & $\mathrm{x}$ & $\mathrm{x}$ & $\mathrm{x}$ & $\mathrm{x}$ & $\mathrm{x}$ & $\mathrm{x}$ & $\mathrm{x}$ & & $\mathrm{x}$ & $\mathrm{x}$ & $\mathrm{x}$ & $\mathrm{x}$ & $\mathrm{x}$ & $\mathrm{x}$ & 18 \\
\hline Web search & & $\mathrm{x}$ & $\mathrm{x}$ & $\mathrm{x}$ & $\mathrm{x}$ & $\mathrm{x}$ & $\mathrm{x}$ & $\mathrm{x}$ & $\mathrm{x}$ & $\mathrm{x}$ & $\mathrm{x}$ & $\mathrm{x}$ & $\mathrm{x}$ & $\mathrm{x}$ & $\mathrm{x}$ & $\mathrm{x}$ & $\mathrm{x}$ & $\mathrm{x}$ & $\mathrm{x}$ & 18 \\
\hline Books & & $\mathrm{x}$ & $\mathrm{x}$ & $\mathrm{x}$ & $\mathrm{x}$ & $\mathrm{x}$ & $\mathrm{x}$ & $\mathrm{x}$ & $\mathrm{x}$ & $\mathrm{x}$ & $\mathrm{x}$ & $\mathrm{x}$ & $\mathrm{x}$ & & $\mathrm{x}$ & $\mathrm{x}$ & $\mathrm{x}$ & $\mathrm{x}$ & $\mathrm{x}$ & 17 \\
\hline Definitions & & $\mathrm{x}$ & $\mathrm{x}$ & & $\mathrm{x}$ & $\mathrm{x}$ & $\mathrm{x}$ & $\mathrm{x}$ & $\mathrm{x}$ & $\mathrm{x}$ & $\mathrm{x}$ & $\mathrm{x}$ & $\mathrm{x}$ & $\mathrm{x}$ & $\mathbf{x}$ & $\mathrm{x}$ & $\mathrm{x}$ & $\mathrm{x}$ & $\mathrm{x}$ & 17 \\
\hline Portals & $\mathrm{x}$ & $\mathrm{x}$ & & $\mathrm{x}$ & $\mathrm{x}$ & $\mathrm{x}$ & & & $\mathrm{x}$ & $\mathrm{x}$ & $x$ & $\mathrm{x}$ & & $\mathrm{x}$ & $\mathbf{x}$ & & $\mathrm{x}$ & $\mathrm{x}$ & $\mathrm{x}$ & 14 \\
\hline Databases & $\mathrm{x}$ & $\mathrm{x}$ & & $\mathrm{x}$ & $\mathrm{x}$ & $\mathrm{x}$ & & $\mathrm{x}$ & & & & & & $\mathrm{x}$ & $\mathbf{x}$ & $\mathrm{x}$ & $\mathrm{x}$ & $\mathrm{x}$ & $\mathrm{x}$ & 12 \\
\hline Teaching files & & $\mathrm{x}$ & $\mathbf{x}$ & & & $\mathrm{x}$ & $\mathrm{x}$ & $\mathrm{x}$ & $\mathrm{x}$ & $\mathrm{x}$ & & $\mathrm{x}$ & & $\mathrm{x}$ & $\mathbf{x}$ & $\mathrm{x}$ & & $\mathrm{x}$ & & 12 \\
\hline 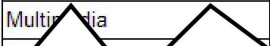 & & & & & & & & $\mathrm{x}$ & & & & $x$ & & & $\mathrm{x}$ & & & $x$ & & 7 \\
\hline
\end{tabular}

Figura 3 - Freqüência dos Contextos nas áreas do conhecimento do OJS/SERUFPR/RST/Context

Fonte: os autores com base na OJS/SER-UFPR/RST/Context.

Em um segundo momento, definiu-se a área de conhecimento de Humanidades (humanities) como a prioritária para o Projeto Piloto em Comunicação e, a partir daí, uma proposta de tradução (do inglês para o português) da terminologia utilizada na ferramenta. Ainda que o contexto "livro" (book) inexista como default na área de humanidades (FIGURA 3), a equipe de trabalho decidiu validá-lo para a etapa de avaliação de fontes. O mesmo ocorreu com os contextos "teses e dissertações", "revisões de livros", "crítica literária", e "textos relacionados". Estas inclusões se respaldaram na relação explícita entre o tipo de contexto e a área de conhecimento definida como piloto para análise, considerando-se as argumentações de MUELLER (2005) e JUANES et alii (2002). O Quadro 1 apresenta a proposta de tradução/adaptação desta terminologia, apoiada na versão 2.1.1 do OJS/SER/UFPR. 


\begin{tabular}{|c|c|c|}
\hline $\begin{array}{l}\text { Contextos mais frequientes } \\
\text { idioma original }\end{array}$ & $\begin{array}{c}\text { Contextos especificos em } \\
\text { Ciências Humanas } \\
\text { idioma original }\end{array}$ & $\begin{array}{l}\text { Contextos específicos em } \\
\text { Ciências Humanas } \\
\text { proposta de tradução }\end{array}$ \\
\hline Author's work's & Author's work's & Outro(s) trabalho(s) do(s) autor(es) \\
\hline Media reports & Media reports & Jornal e rádio \\
\hline Online forums & Online forums & Fóruns/Listas de discussão \\
\hline Pay-per-view & Pay-per-view & Pay-per-view \\
\hline Government policy & Government policy & Politicas governamentais \\
\hline Related studies & Related studies & Estudos relacionados \\
\hline Web search & Web search & Buscadores da internet \\
\hline Books & Books & Livros \\
\hline Definitions & Definitions & Defínição de termos \\
\hline Portals & Portals & Portais \\
\hline Databases & Databases & Bases de dados \\
\hline \multirow[t]{5}{*}{ Teaching files } & Teaching files & Material didático \\
\hline & Dissertations & Teses e Dissertações \\
\hline & Book reviews & Revisões de livros \\
\hline & Literacy critics & Critica literária \\
\hline & Related texts & Textos relacionados \\
\hline
\end{tabular}

Quadro 1 - Proposta de tradução/adaptação da terminologia da OJS/SER-UFPR/RST/Context

Fonte: os autores com base na OJS/SER-UFPR/RST/Context.

O passo seguinte foi o de análise de qualidade das fontes de informação inseridas em cada contexto a partir do diagrama representado na FIGURA 2 previamente apresentada. Em um primeiro momento, gerou-se um arquivo de texto com todas as fontes default existentes no OJS/SER-UFPR, filtrando-se aquelas relacionadas com a área de Humanidades e incluindo-se os demais contextos considerados como válidos pela equipe de pesquisadores. Em seguida, elaborou-se uma planilha no MS/Excel contendo os campos: título, URL, string (de pesquisa), descrição, descrição traduzida/adaptada, área $\mathrm{CNPq}$, avaliação (se mantida, mantida com ressalvas, ou descartada) e observações. Foram analisadas oitenta e quatro fontes existentes nos contextos e área definida (Quadro 2) e, após a avaliação, trinta e seis foram mantidas, representando $43 \%$ do total. 


\begin{tabular}{|c|c|c|c|c|}
\hline Contexto traduzido & Descrição & $\begin{array}{l}\text { Fontes listadas } \\
\text { pelo OJS/SER }\end{array}$ & $\begin{array}{l}\text { Fontes } \\
\text { mantidas após } \\
\text { avaliação }\end{array}$ & $\begin{array}{l}\% \text { das fontes } \\
\text { mantidas }\end{array}$ \\
\hline $\begin{array}{l}\text { Outro(s) trabalho(s) } \\
\text { do(s) autor(es) }\end{array}$ & $\begin{array}{l}\text { Identifica outros trabalhos do(s) autor(es) } \\
\text { em demais repositórios de acesso aberto }\end{array}$ & 8 & 5 & $63 \%$ \\
\hline Jornal e rádio & $\begin{array}{l}\text { Identifica os principais jomais e rádios } \\
\text { disponiveis gratuitamente na Internet }\end{array}$ & 9 & 2 & $22 \%$ \\
\hline $\begin{array}{l}\text { Fóruns/Listas de } \\
\text { discussão }\end{array}$ & $\begin{array}{l}\text { Identifica fóruns ou listas de discussão } \\
\text { disponiveis online }\end{array}$ & 3 & 2 & $67 \%$ \\
\hline Pay-per-view & $\begin{array}{l}\text { Identifica serviços pagos de informação } \\
\text { (especialmente de artigos cientificos) }\end{array}$ & 4 & 4 & $100 \%$ \\
\hline $\begin{array}{l}\text { Politicas } \\
\text { governamentais }\end{array}$ & $\begin{array}{l}\text { Identifica portais e outros serviços; } \\
\text { repositórios de informação online de } \\
\text { responsabilidade governamental }\end{array}$ & 7 & 2 & $29 \%$ \\
\hline Estudos relacionados & - & 10 & 1 & $10 \%$ \\
\hline Buscadores da Internet & $\begin{array}{l}\text { Identifica ferramentas gratuitas de busca } \\
\text { na Internet }\end{array}$ & 3 & 2 & $67 \%$ \\
\hline Livros & $\begin{array}{l}\text { Identifica outros trabalhos do(s) } \\
\text { mesmo(s) tema(s) em livros (texto } \\
\text { completo ou apenas referências) } \\
\text { disponiveis em repositórios/bases de } \\
\text { dados gratuitas }\end{array}$ & 4 & 2 & $50 \%$ \\
\hline Definição de termos & $\begin{array}{l}\text { Identifica definições de termos existentes } \\
\text { no texto em dicionários, enciclopédias e } \\
\text { outras fontes de acesso gratuito }\end{array}$ & 7 & 6 & $86 \%$ \\
\hline Portais & $\begin{array}{l}\text { Identifica Portais da Web na área de } \\
\text { conhecimento da revista }\end{array}$ & 6 & - & $0 \%$ \\
\hline Bases de dados & $\begin{array}{l}\text { Identifica outros trabalhos do(s) autor(es) } \\
\text { em bases de dados de resumos e/ou } \\
\text { textos completos de acesso gratuito }\end{array}$ & 6 & 1 & $17 \%$ \\
\hline Material didático & $\begin{array}{l}\text { Identifica material de acesso livre } \\
\text { produzido para fins didáticos }\end{array}$ & 1 & 1 & $100 \%$ \\
\hline Teses e Dissertações & $\begin{array}{l}\text { Identifica repositorios institucionais e de } \\
\text { acesso gratuito de teses e dissertações }\end{array}$ & 2 & 1 & $50 \%$ \\
\hline Revisões de livros & $\begin{array}{l}\text { Identifica revisões de livros e de demais } \\
\text { textos das áreas de ciências humanas e } \\
\text { literatura }\end{array}$ & 5 & 4 & $80 \%$ \\
\hline Crítica literária & $\begin{array}{l}\text { Identifica material de acesso gratuito } \\
\text { relacionado à critica literária e material } \\
\text { biográfico }\end{array}$ & 1 & 1 & $100 \%$ \\
\hline \multirow[t]{2}{*}{ Textos relacionados } & - & 8 & 2 & $25 \%$ \\
\hline & TOTAIS & 84 & 36 & $43 \% *$ \\
\hline
\end{tabular}

Quadro 2 - Resultados da avaliação das fontes constantes dos contextos da área Humanidades Fonte: os autores com base na OJS/SER-UFPR/RST/Context.

Liinc em Revista, v.4, n.2, setembro 2008, Rio de Janeiro, p. 253-268 - http://www.ibict.br/liinc 
* porcentagem relativa ao total de fontes avaliadas

Tendo sido aplicado o diagrama com as dimensões de qualidade para fontes de informação, percebeu-se que a permanência ou não do recurso, independentemente de seu contexto, se definia especialmente na dimensão de conteúdo. Nesta perspectiva, o descarte do recurso ocorreu sob as seguintes condições: a) público-alvo da fonte não afeto à área de Humanidades; b) veiculação de anúncios no site; e c) dificuldade de se identificarem os objetivos pretendidos pelos criadores do recurso; e d) atualidade (data de criação/ atualização). Destaca-se o fato de que algumas fontes default, ainda que arroladas na área de Humanidades, não lhe são restritas. Tal característica não justificaria por si só o descarte, porém implica em uma discussão sobre as condições de agregação de recursos multidisciplinares sob uma fonte "guarda-chuva". Pari passu, o fato de algumas fontes aparecerem em mais de um contexto exigirá análise detalhada do seu efetivo conteúdo, de forma a embasar um possível descarte ou o reconhecimento formal da permanência deste nos diferentes contextos identificados.

Outros critérios para o expurgo de algumas fontes no universo pesquisado referem-se à dimensão de autoria. Isto é, foram descartados sites ou páginas web cuja autoria (individual, coletiva/institucional) não foi identificada. O mesmo ocorreu com fontes cujos direitos autorais não estavam explícitos (dimensão do recurso). Foram desconsideradas fontes com links desativados (variável de permanência/estabilidade). Uma situação interessante surgiu quando da análise das fontes nos contextos "estudos relacionados" e "textos relacionados". Ainda que 10\% e $25 \%$ de fontes foram aproveitadas, respectivamente, a equipe de pesquisadores optou por não descrevê-los e, eventualmente, retirá-los da ferramenta, uma vez que tais fontes aparecem em outros contextos.

A dimensão dos repositórios provavelmente gerará controvérsia na sequiência do estudo, pois, na medida em que se procedeu à avaliação das fontes, verificou-se que outras poderiam vir a substituir aquelas expurgadas. Porém, parte significativa destas "novas" fontes não provém de repositórios de acesso aberto, o que atinge o centro da proposta do movimento de amplo e livre acesso à informação científica.

\section{Considerações Parciais e Continuidade do Projeto}

Outra atividade no atual estágio do projeto é a descrição propriamente dita das fontes e a escolha de outras a serem integradas na ferramenta. Para a retirada e a inclusão das fontes de informação presentes na RST/Context, além dos critérios de qualidade, estabeleceram-se procedimentos para normalização de resumos indicativos. Tais procedimentos apoiaram-se em elementos descritivos de fontes de informação apresentados no estudo desenvolvido por Cendón (2001) (Quadro 3): 


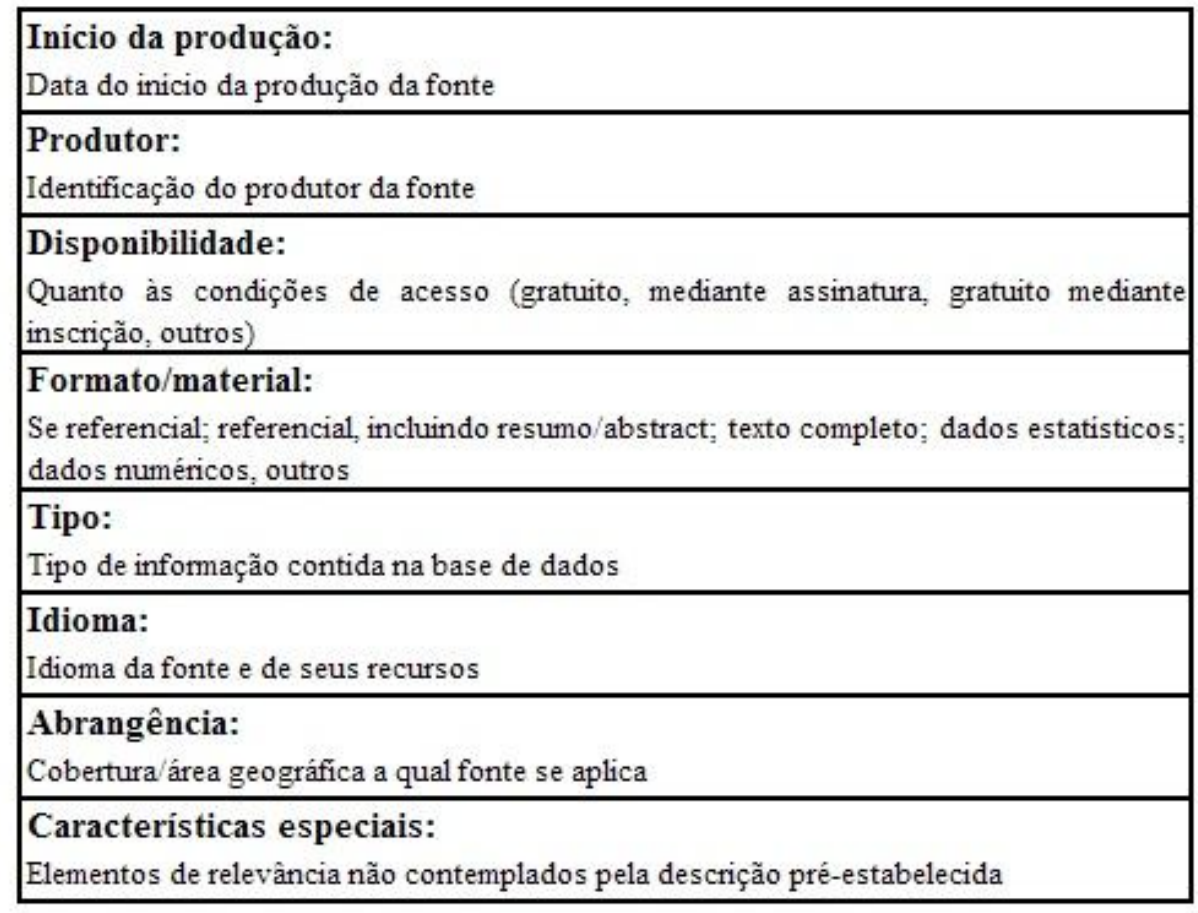

Quadro 3 - Elementos para descrição de fontes de informação

Fonte: Adaptado de: CENDÓN, B. V. Fontes eletrônicas de informação para negócios: formato utilizado para a descrição das bases de dados. 2001. Disponível em: 〈http://www.eci.ufmg.br/cendon/pesquisa/descricao.htm〉.

Tais elementos originaram o seguinte roteiro de descrição das fontes:

\begin{tabular}{l} 
Fonte "X" \\
\hline "Disponibilizado/distribuido/criado... desde [Data do inicio da produção da base de \\
dados] pelo(a) [Nome do produtor]. Oferece [gratuitamente, mediante assinatura, \\
gratuitamente mediante inscrição] o que, em que formato/material [referencial; \\
referencial, incluindo resumo/abstract; texto completo; dados estatisticos; numéricos; \\
arquivos...] no idioma [inserir idioma]. Tem abrangência [internacional; nacional \\
(qual); regional(qual); estadual (qual); institucional (qual)]. Tem como características \\
especiais [montar lista de tais caracteristicas]."
\end{tabular}

Quadro 4 - Roteiro de descrição das fontes do OJS/SER-UFPR/RST/Context

Fonte: os autores com base na OJS/SER-UFPR/RST/Context. 
A título de ilustração, exemplifica-se abaixo uma das descrições já elaboradas para uma das fontes que permanecerá na RST/Context

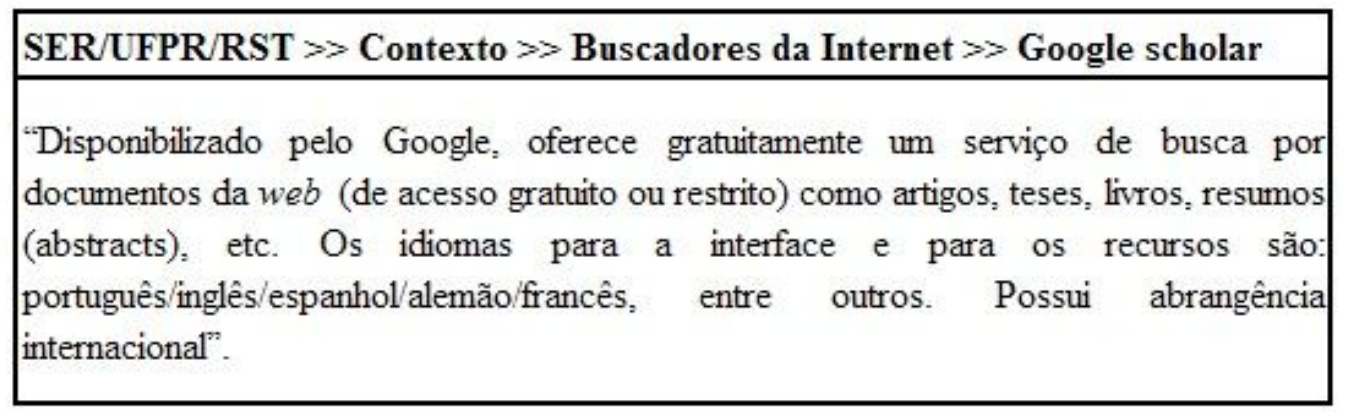

\section{Quadro 5 - Exemplo de descrição para fonte da OJS/SER-UFPR/RST/Context}

Fonte: os autores com base na OJS/SER-UFPR/RST/Context.

Todas as fontes validadas para o Piloto foram descritas com base neste modelo. Além disto, outros desafios para o Projeto se concentram, no momento, na revisão das strings de pesquisa (devido ao significativo número de links "quebrados") e em localizar fontes em língua portuguesa compatíveis com as funções identificadas nas fontes default que, em sua maioria, estão em língua inglesa.

Sem sombra de dúvida o movimento de arquivos abertos e suas ferramentas vêm alterando a dinâmica política e operacional da comunicação científica tradicional. Como toda inovação, a proposta tem suas complexidades, que não deixam de ser variações daquelas identificadas anteriormente por CAMPELLO (1988, 1998, 2000) e PEIXOTO (2002). A proliferação de fontes em formato eletrônico tornou a explosão bibliográfica/informacional ainda mais aguda e o risco não é apenas da obsolescência, mas do próprio desconhecimento do conjunto "ideal" de informações disponíveis frente ao universo sempre crescente de recursos. A projeção de PEIXOTO (2002) quanto à preferência da comunidade de pesquisadores pelo uso de informações obtidas pelos recursos computacionais se confirma, assim como permanecem as barreiras de idioma, a dispersão em diferentes vetores e a dificuldade de se garantir confiabilidade ao recurso. Porém, as tecnologias permitem um fluxo de comunicação ágil entre pesquisadores, assim como a redução significativa no custo de organização, distribuição e manutenção de fontes disponíveis em rede.

Quanto ao item confiabilidade, pretende-se aprofundar as discussões que envolvem os elementos descritos no diagrama de dimensões de qualidade de fontes de informação e, a partir daí, selecionar outras fontes para expandir o conteúdo dos contextos validados nesta fase. O passo seguinte será transportar este conjunto de informações para o software ele mesmo, efetivar testes de consistência e avaliar o Projeto Piloto a partir do uso por um grupo definido. Pretende-se, em médio prazo, oferecer a experiência como benchmaking para as demais revistas que integram o 
PERIÓDICOS DA UFPR/SER ${ }^{1}$ e demais interessados na discussão do OJS/SEER.

Artigo recebido em 30/05/2008 e aprovado em 01/07/2008.

\section{Referências}

BIREME. Metodologia LIS: Critérios para seleção de fontes de informação em saúde disponíveis na Internet (revisada). (C) 2005. Disponível em: <http://lis.bvs.br/lisRegional/P/Metodologia/CriteriosPortuguesfinal.doc>. Acesso em: abr. 2008.

CAMPELlO, Bernadete Santos, CAMPOS, Carlita Maria. Fontes de informação especializada: características e utilização. Belo Horizonte: UFMG/PROED, 1988. 44 p.

CAMPELLO, Bernadete Santos; CALDEIRA, Paulo da Terra; MACEDO, Vera Amália Amarante. Formas e expressões do conhecimento: introdução às fontes de informação. Belo Horizonte : Escola de Biblioteconomia da UFMG, 1998. 413 p.

CAMPELLO, Bernadete Santos; CENDÓN, Beatriz Valadares; KREMER, Jeannete Marguerite (org.). Fontes de informação para pesquisadores e profissionais. Belo Horizonte : Ed. UFMG, 2000. 319 p.

CENDÓN, Beatriz Valadares. Fontes eletrônicas de informação para negócios: formato utilizado para a descrição das bases de dados. 2001. Disponível em: <http://www.eci.ufmg.br/cendon/pesquisa/descricao.htm>. Acesso em: 29 jun. 2007.

JUANES, Ivone A. Soares; KYIOTANI, Normanda Miranda; NORONHA, Daisy Pires. Produção científica em comunicação dos docentes da ECA/USP. In: Congresso Brasileiro de Ciências da Comunicação, 25, 2002, Salvador. Anais... São Paulo: Intercom, 2002. Disponível em: < http://reposcom.portcom.intercom.org.br/handle/1904/19267>. Acesso em: 5 jul. 2007.

KIRK, Elizabeth E. Evaluating Information Found on the Internet. (C1996. Disponível em: <http://www.library.jhu.edu/researchhelp/general/evaluating/index.html>. Acesso em: abr. 2008.

MUELLER, Suzana Pinheiro Machado. A publicação da ciência: áreas científicas e seus canais preferenciais. Datagramazero, v. 6, n. 1, fev./2005. Disponível em: <http://www.datagramazero.org.br/fev05/Art_02.htm\#Autor>. Acesso em: 5 jul. 2007.

ORMONDROYD, Joan. Critically analyzing information sources. (C2004. Disponível em: <http://www.library.cornell.edu/olinuris/ref/research/skill26.htm>. Acesso em: abr. 2008. (Atualizado, editado e transposto para a Web por Michael Engle e Tony Cosgrave)

\footnotetext{
${ }^{1} \mathrm{http} / / /$ www.prppg.ufpr.br/stricto_sensu/periodicos.html
} 
PEIXOTO, M. P. Fontes de estudo na escola médica : recursos instrucionais utilizados por alunos de Medicina na Região Sudeste do Brasil. Revista Brasileira de Educação Médica, Rio de Janeiro, v. 26, n. 1, jan./abr. 2002.

RIEH, Soo Young . Cognitive Authority. In: K.E. Fisher, S. Erdelez, \& E. F. McKechnie (Eds.), Theories of information behavior: A researchers' guide. Medford, NJ: Information Today, p. 8387. 2005. Disponível em: 〈http://www.si.umich.edu/rieh/papers/rieh_IBTheory.pdf〉. Acesso em: abr. 2008.

RIEH, Soo Young; BELKIN, Nicholas J. Interaction on the Web: Scholars' Judgment of Information Quality and Cognitive Authority. Annual Meeting of the ASIS, 63rd, Proceedings, 2000, pp. 25-38. Disponível em: 〈http://newweb2.si.umich.edu/rieh/papers/rieh_asis2000.pdf〉. Acesso em: abr. 2008.

RIEH, Soo Young, BELKIN, Nicholas J. Understanding Judgment of Information Quality and Cognitive Authority in the WWW. Annual Meeting of the American Society for Information Science, 61st, Proceedings 35, pp. 279-289. 1998. Disponível em: <http://www.si.umich.edu/rieh/papers/asis98.pdf>. Acesso em: abr. 2008.

STUMPF, Ida Regina Chitto. Avaliação das revistas de comunicação pela comunidade acadêmica da área. Em Questão, Porto Alegre, v. 9, n. 1, p. 25-38, jan./jun. 2003. Disponível em: <http://www.seer.ufrgs.br/index.php/EmQuestao/article/view/57/17>. Acesso em: 5 jul. 2007.

TILLMAN, Hope N. Evaluating Quality on the Net. 2003. Disponível em: <http://www.hopetillman.com/findqual.html>. Acesso em: abr. 2008.

WILLINSKY, John. As open access is public access, can journals help policymakers read research? Canadian Journal of Communication, v. 29, n. 3-4, 2004, p. 381-401.

WILLINSKY, John. Opening Access: Reading (Research) in the Age of Information. In: C.M. Fairbanks, J. Worthy, B. Maloch, J.V. Hoffman, and D.L. Schallert (editors). National Reading Conference Yearbook, 51. Oak Creek, Wis.: National Reading Conference, pp. 32-46, 2003. Disponível em: <http://pkp.sfu.ca/files/NRC_Galley.pdf>. Acesso em: 12 abr. 2008. 